\title{
Mechanical effect of van der Waals interactions observed in real time in an ultracold Rydberg gas
}

\author{
T. Amthor * M. Reetz-Lamour, S. Westermann, J. Denskat, and M. Weidemüllerit \\ Physikalisches Institut, Universität Freiburg, Hermann-Herder-Str. 3, 79104 Freiburg, Germany
}

(Dated: July 7, 2021)

\begin{abstract}
We present time-resolved spectroscopic measurements of Rydberg-Rydberg interactions in an ultracold gas, revealing the pair dynamics induced by long-range van der Waals interactions between the atoms. By detuning the excitation laser, a specific pair distribution is prepared. Penning ionization on a microsecond timescale serves as a probe for the pair dynamics under the influence of the attractive long-range forces. Comparison with a Monte Carlo model not only explains all spectroscopic features but also gives quantitative information about the interaction potentials. The results imply that the interaction-induced ionization rate can be influenced by the excitation laser. Surprisingly, interaction-induced ionization is also observed for Rydberg states with purely repulsive interactions.
\end{abstract}

PACS numbers: 32.80.Rm, 34.20.Cf, 34.10.+x, 34.60.+z

Long-range dipolar interactions ubiquitously appear in nature as the cause for binding forces ranging from atomic and molecular gases [1] all the way to large biological systems 2]. Rydberg atoms have attracted much interest in this context, as they represent an ideal system to study quantum dynamics under the influence of dipolar interactions. As a prominent example from cavity quantum electrodynamics, attractive forces between Rydberg atoms and conducting surfaces have been observed as level shifts in atomic beam experiments [3]. RydbergRydberg interactions leading to resonant energy transfer have been studied in thermal beam experiments [4] and later in ultracold Rydberg gases [5, 6, 7]. The longrange dipolar interactions can be used to block multiple Rydberg excitation [8, 9] and to create many-particle entangled states, which may be employed for quantum information processing [10, 11]. So far, investigations of Rydberg-Rydberg interactions have mainly focused on the electronic degrees of freedom neglecting the center-ofmass motion ("frozen Rydberg gas" 7]]). In this Letter, we present real-time measurements of the motion of interacting pairs of Rydberg atoms revealing the character and strength of the long-range interparticle interactions.

In most molecular and atomic systems the relevant timescales of interparticle dynamics are in the sub-ns regime calling for very fast probes. Ultracold atoms bridge to the ns regime as the thermal energy $(T<1 \mathrm{mK})$ is negligible which allows one to study systems with much weaker interactions or large interatomic distances. Due to the negligible kinetic energy, the dynamics of the gas is fully determined by the interatomic interactions. Interactions in a cold atomic sample have been studied time-resolved in the case of ground state atoms using a pump-probe scheme 12 allowing for coherent control of the collision process [13]. Evidence for interactioninduced motion in cold Rydberg gases was recently found spectroscopically as the cause for Penning ionization 14]. By combining time-resolved and spectroscopic measure-
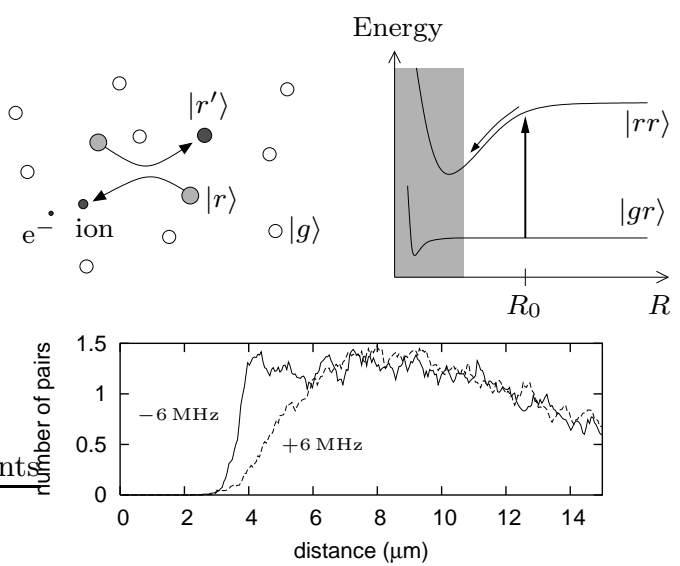

FIG. 1: Excitation and interaction-induced ionization of a pair of atoms on an attractive vdW potential. The atoms are initially excited to state $|r\rangle$ at a distance $R_{0}$ by a slightly reddetuned laser. After excitation they are accelerated towards each other and collide, leading to ionization of one of the atoms. The region where this Penning ionization can occur is indicated in gray. The number of pairs excited at specific distances is shown in the lower graph for different detunings.

ments, we quantitatively examine the van der Waals (vdW) interactions between two Rydberg atoms. Our measurements can be directly compared to calculations of induced-dipole interaction potentials for two Rydberg atoms, which have so far only been performed neglecting spin-orbit coupling [15]. These interaction potentials may even bear bound states resulting in the formation of exotic ultralong-range molecules [16].

The idea of the experiment is schematically illustrated in Fig. 1 The vdW interaction between two Rydberg atoms leads to an energy shift of the pair excitation which depends on the pair distance. A red-detuned laser will therefore preferably excite atoms on an attractive potential in a certain range of distances around an already excited atom. In this way one can use the laser detuning 
to create a sample with a specific distribution of pair distances. Two examples for $+6 \mathrm{MHz}$ (blue) and $-6 \mathrm{MHz}$ (red) detuning as derived from our model are also plotted in Fig. 1 Once a pair of Rydberg atoms is excited on an attractive potential, the atoms will be accelerated towards each other and collide after a certain time depending on their initial distance $R_{0}$. These collisions can lead to Penning ionization. By measuring the number of ions produced after a variable time $\Delta t$ one can follow the dynamics of the system in real time. The ionization is thus used as a monitor signal for pair dynamics giving quantitative information about the interaction strength.

In our setup we trap ${ }^{87} \mathrm{Rb}$ atoms at temperatures below $100 \mu \mathrm{K}$ and a peak density on the order of $10^{10} \mathrm{~cm}^{-3}$ in a magneto-optical trap (MOT). The atoms are then excited to Rydberg states using a two-photon excitation scheme. The two atomic transitions $5 \mathrm{~S}_{1 / 2} \rightarrow 5 \mathrm{P}_{3 / 2}$ and $5 \mathrm{P}_{3 / 2} \rightarrow \mathrm{n} \ell$ are realized with two $\mathrm{cw}$ laser systems at $780 \mathrm{~nm}$ and $480 \mathrm{~nm}$, respectively. The frequency of the 480-nm excitation laser is actively stabilized using an ultra-stable reference cavity. The 480 -nm laser is focused to a waist of $\sim 37 \mu \mathrm{m}$ at the center of the MOT with a power of $10 \mathrm{~mW}$. Two metal grids are used to apply electric fields for stray field compensation and for field ionization of the Rydberg states. Ions are detected on a microchannel plate detector. Ref. 17] describes the setup in more detail.

The experimental cycle, repeated every $70 \mathrm{~ms}$, is as follows: The excitation laser is switched on for $100 \mathrm{~ns}$ at a given detuning. This time has been chosen sufficiently short so that the movement of the atoms is negligible and no ionization takes place during the excitation. The gas can then evolve freely for a variable time $\Delta t$. After that, an electric field ramp is applied to field ionize the Rydberg atoms and accelerate the ions towards the detector. Ions produced by collisions will be drawn to the detector at the very beginning of the ramp (i.e. at low accelerating fields), while Rydberg states ionize at a finite electric field, generating a delayed detector signal. Using two boxcar integrators, the two signals are recorded simultaneously.

Fig. [2]shows the Rydberg and ion signals at the $60 \mathrm{D}_{5 / 2}$ resonance as a function of the excitation laser frequency. The frequency axis is centered at the atomic resonance. In Fig. 2a the Rydberg signal measured directly after excitation is displayed. The width of the excitation line is given by the width of the intermediate $5 \mathrm{P}$ level $(6 \mathrm{MHz}$ FWHM), the laser linewidths (2-5 MHz FWHM), the finite excitation time (resulting in a $4.2 \mathrm{MHz}$ FWHM of the Fourier transform), and a $\sim 2 \mathrm{MHz}$ broadening due to residual electric fields. The line shape should thus be a Voigt profile, but it is still described reasonably well by a Lorentz fit (also shown in the graph) yielding a FWHM of $12.5 \mathrm{MHz}$ as expected from the above numbers. In the graphs in Fig. 20 the development of the ion signal after different interaction times $\Delta t$ is shown. All data are (a)
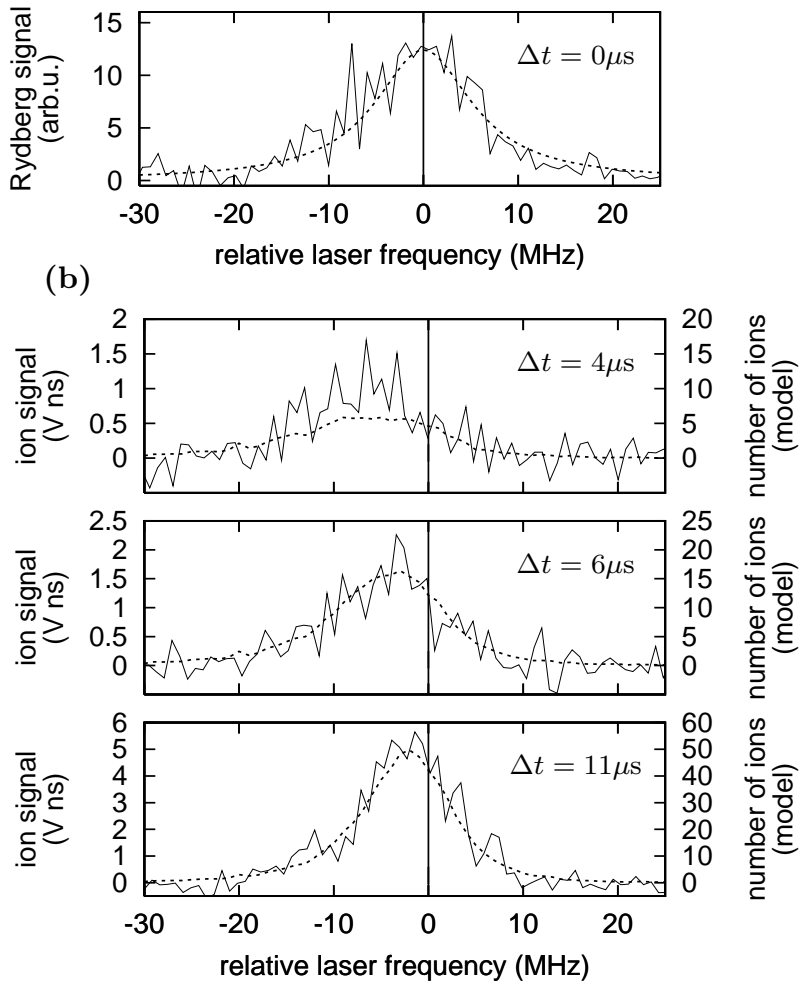

FIG. 2: (a) $60 \mathrm{D}_{5 / 2}$ Rydberg excitation line (solid), with fitted Lorentzian (dotted), (b) development of the ion signal for different interaction times (solid) compared to Monte-Carlo simulation (dotted). The laser frequency is given relative to the atomic resonance.

compared to the results of the simulation (dotted lines) described below. Within several $\mu$ s the number of ions increases steadily. The ion signal peaks at the red-detuned side of the atomic resonance and exhibits a pronounced red wing. As the interaction time increases, most ions appear nearer to the atomic resonance, which can be observed as a shift of the ionization line towards zero detuning. These spectral features can easily be understood in the picture of colliding pairs: After a short interaction time only very close pairs will have had the time to collide, and these pairs are preferably excited at large detuning. For very long times, the line shape resembles the initial Rydberg excitation line, as almost all atoms are ionized. From these results it is obvious that by slight detuning of the excitation laser the initial ionization rate can be controlled. This can be of importance for applications like quantum computation with Rydberg atoms, where the presence of ions acts as a decoherence process, and for the investigation of ultracold plasma formation.

While a simple analytical description of pair excitations as in Ref. 18] can qualitatively explain the behavior, it turns out to be insufficient to describe the complex excitation process and thus does not provide quantitative information about the interaction potentials. Instead 
of considering only the excitation of individual pairs far away from each other our model accounts for the interaction between all atoms and allows for clustering of Rydberg excitation around already excited atoms in order to describe the system more realistically and to reproduce the measured line shapes. We have therefore performed a Monte Carlo simulation. 100000 atoms are randomly placed in a box with a Gaussian distribution along the excitation laser axis to represent the MOT with a peak density of $1 \times 10^{10} \mathrm{~cm}^{-3}$. Excitation of the gas is described by an iterative procedure: In each iteration step, each of the atoms can be excited with a certain probability. For atom $i$ it is given by $P_{i} \propto \mathcal{L}\left(\delta-V\left(R_{i}\right)\right) \exp \left(-2\left(x_{i}^{2}+y_{i}^{2}\right) / w^{2}\right)$. $\mathcal{L}$ is the line profile representing the $12.5 \mathrm{MHz}$ linewidth of the Rydberg excitation as described above. $\mathcal{L}$ is evaluated at a detuning consisting of the laser detuning $\delta$ and the interaction energy $V\left(R_{i}\right)$ with the nearest Rydberg neighbor to atom $i$. The Gaussian intensity distribution of the excitation laser with waist $w=37 \mu \mathrm{m}$ is accounted for by the last factor. After each Rydberg excitation, the table of nearest Rydberg neighbors is recalculated for all atoms. This excitation process is iterated to yield the actual excitation fraction of a few percent at the center of the MOT.

The excited atoms are then combined to disjoint pairs by successively choosing the two atoms closest to each other. From the resulting list of distances, shown as a histogram in the lower graph in Fig. 11 the number of pairs colliding until a certain time $\Delta t$ can be determined. We assume an attractive potential of the form $V(R)=-C_{6} / R^{6}$. The time it takes a pair of atoms with initial distance $R_{0}$ and an initial velocity of zero to collide can then be approximated by the time to reach a distance of zero, $\tau_{\text {coll }} \approx 0.2156 R_{0}^{4}\left(2 C_{6} / m_{\mathrm{Rb}}\right)^{-1 / 2}[18]$. To determine the number of ions produced until a time $\Delta t$, we count the pairs that collide within this time. The whole simulation is performed for different laser detunings to obtain the line shape of the ion signal.

The resulting traces shown in Fig. 2] are averaged over ten random realizations as described above. The best agreement of the Monte Carlo simulation with the experimental results is found for $C_{6}=2 \times 10^{20} \mathrm{au}$, while values below $10^{20}$ and above $10^{21}$ are not compatible with the measured spectra. This is in agreement with calculations following Ref. [15]. Note that in the actual experiment an effective potential is observed, which averages over all possible molecular symmetries. The measured ionization signals (Fig. 20) are well reproduced by the simulation.

While this spectroscopic view shows how the line shape is reproduced by the simulation, one can gain some more insight in the system by comparing the time development of the measured signal and the model at specific detunings, as displayed in Fig. 3. The model predicts a threshold for the ion production at around 3-4 $\mu \mathrm{s}$, which is also observed in the experiment. Furthermore, when comparing the ionization rates at different detunings rel-

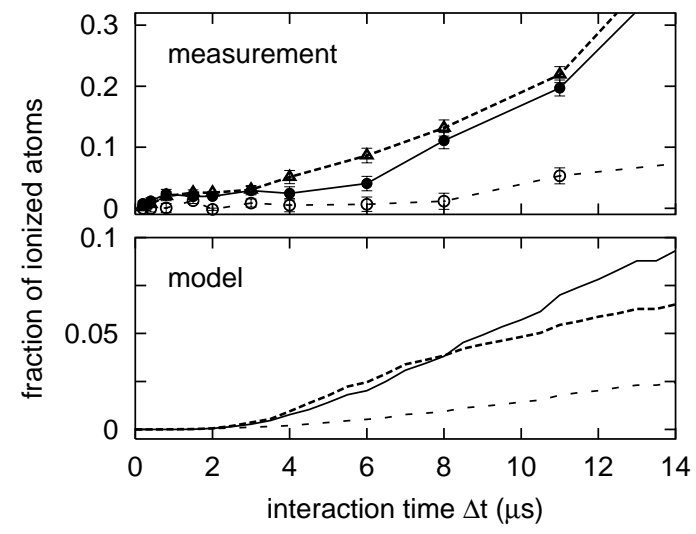

FIG. 3: Signal growth at different detunings: $0 \mathrm{MHz}$ (solid), $+6 \mathrm{MHz}$ (dotted), $-6 \mathrm{MHz}$ (dashed). Upper graph: measured data (data points averaged over $3 \mathrm{MHz}$ ), lower graph: model. The scale of the measurement data contains a systematic error of a factor of two.

ative to each other, we find very similar behavior of model and experiment: At $-6 \mathrm{MHz}$ the rate is comparable to the one at zero detuning, while at $+6 \mathrm{MHz}$ the rate is significantly lower. The error bars at the measured data points display the standard deviation of fluctuations in the signal, while the scale is subject to a systematic error of a factor of two.

So far we have considered the interaction potential to be of pure vdW type. However, as pointed out in Ref. [14], nearby dipole-coupled pair states introduce a $-1 / R^{3}$ behavior of the interaction potential for small distances. To estimate the influence of this effect we have used a simple interaction Hamiltonian $\mathcal{H}$ considering only the strongest couplings. From our Monte Carlo model we infer that the distance between pairs of atoms is always above $3 \mu \mathrm{m}$ (see Fig. 11). These distances are above the $\mathrm{vdW}$ radius (the distance at which the $-1 / R^{3}$ behavior becomes apparent), i.e. the eigenenergies of $\mathcal{H}$ do not differ significantly from a pure $-C_{6} / R^{6}$ potential with $C_{6}=5.4 \times 10^{20} \mathrm{au}$. Even when comparing the collision times of pairs derived from these potentials we find only slight differences for typical initial distances. We thus expect a description in terms of a vdW potential to be appropriate. In contrast, when re-running the Monte Carlo calculations considering only the $-1 / R^{3}$ component of the potential, the ion signal line shape could not be reproduced at all.

This description will only be applicable as long as cold Rydberg-Rydberg collisions are the main cause of ionization. As soon as the ion density becomes sufficiently large to trap electrons, electron-Rydberg collisions will determine the dynamics of the system, causing ionization as well as $\ell$ and $\mathrm{n}$ mixing [19]. Redistribution of atoms to other Rydberg states by Penning ionization or by black-body radiation (estimated rates below $5600 \mathrm{~s}^{-1}$ [20]) as well as direct black-body ionization will also af- 


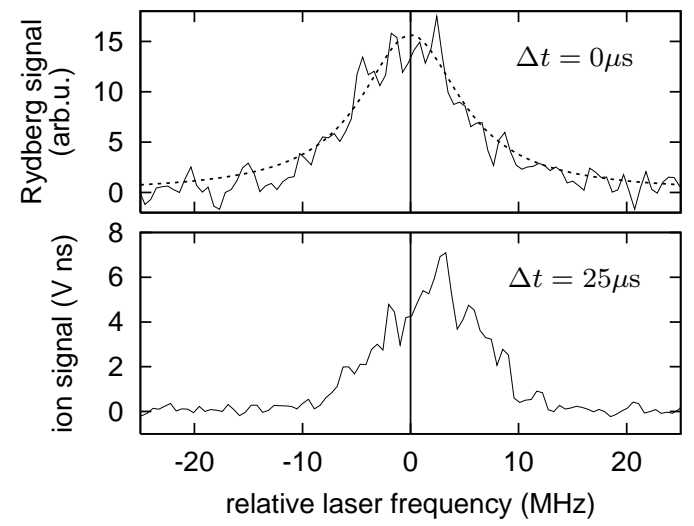

FIG. 4: 60S Rydberg excitation line (upper graph) and preionized atoms after $25 \mu \mathrm{s}$ interaction time (lower graph). The ions are produced on the blue-detuned side of the resonance. Excitation time is $300 \mathrm{~ns}$, and excitation laser power $22 \mathrm{~mW}$. The graphs have different signal height scales. The fit in the upper graph (dotted) yields a line width of $11.3 \mathrm{MHz}$, similar to the $60 \mathrm{D}_{5 / 2}$ line.

fect the dynamics of the system, but should not have significant influence during the first few $\mu \mathrm{s}$. For long interaction times, however, we expect our model to become increasingly inaccurate and the measured signal to exceed the simulated one. This is indeed what we observe for $\Delta t>11 \mu \mathrm{s}$ (see Fig. 3).

According to the above analysis one would not expect to see ionization due to inelastic processes when the interaction is repulsive. We have also performed similar measurements for the $60 \mathrm{~S}$ state, which exhibits repulsive vdW interaction [15]. Surprisingly, we still observe ionization, yet only on much longer timescales. Most ions are produced on the blue-detuned side of the resonance, which implies that the ionization rate is in conjuction with the repulsive potential. A possible explanation is the redistribution of states due to black-body radiation leading to attractive dipole-dipole potentials. Atoms initially excited at small distance (i.e. at blue detuning) could then be the first to collide 21]. This process is more difficult to model for a cloud of atoms and a simple approach of including black-body redistribution and subsequent $1 / R^{3}$ attraction in our Monte Carlo model could not explain the observed line shapes. There may also exist attractive pair potentials of other states crossing the repulsive vdW potentials. Atoms initially repelled from each other may reach a crossing and return on the attractive path. We will further investigate this process in future experiments.

In conclusion, the time-resolved spectroscopic measurements reveal the motion of Rydberg atoms under the influence of van der Waals interactions as the cause of Penning ionization. The temporal evolution of the ionization signal allows one to distinguish van der Waals from resonant dipole interactions. The measured strength of the interaction is in good quantitative agreement with theoretical predictions. The accuracy of determining the interaction strength could be increased by using two excitation laser pulses at different frequencies: The first one to excite a dilute Rydberg gas on the atomic resonance, the second one detuned from resonance to create well-defined pair distributions over a larger range of accessible detunings. Förster resonances may be used to switch the character of the interaction from van der Waals to resonant dipole interaction. In this way our experimental method will allow for a more precise determination of long-range potentials and challenge refined models of many-body dynamics under the influence of dipolar forces.

The project is supported in part by the Landesstiftung Baden-Württemberg in the framework of the "Quantum Information Processing" program, and a grant from the Ministry of Science, Research and Arts of BadenWürttemberg (Az: 24-7532.23-11-11/1). We thank T. F. Gallagher and P. Gould for inspiring discussions.

* thomas.amthor@physik.uni-freiburg.de

$\dagger$ m.weidemueller@physik.uni-freiburg.de

[1] L. Santos, G. V. Shlyapnikov, P. Zoller, and M. Lewenstein, Phys. Rev. Lett. 85, 1791 (2000).

[2] K. Autumn, Y. A. Liang, S. T. Hsieh, W. Zesch, W. P. Chan, T. W. Kenny, R. Fearing, and R. J. Full, Nature 405, 681 (2000).

[3] V. Sandoghdar, C. I. Sukenik, E. A. Hinds, and S. Haroche, Phys. Rev. Lett. 68, 3432 (1992).

[4] K. Safinya, J. Delpech, F. Gounand, W. Sandner, and T. Gallagher, Phys. Rev. Lett. 47, 405 (1981).

[5] S. Westermann, T. Amthor, A. L. de Oliveira, J. Deiglmayr, M. Reetz-Lamour, and M. Weidemüller, Eur. Phys. J. D, DOI: 10.1140/epjd/e2006-00130-3 (2006).

[6] W. R. Anderson, J. R. Veale, and T. F. Gallagher, Phys. Rev. Lett. 80, 249 (1998).

[7] I. Mourachko, D. Comparat, F. de Tomasi, A. Fioretti, P. Nosbaum, V. Akulin, and P. Pillet, Phys. Rev. Lett. 80, 253 (1998).

[8] D. Tong, S. Farooqi, J. Stanojevic, S. Krishnan, Y. Zhang, R. Côté, E. Eyler, and P. Gould, Phys. Rev. Lett. 93, 063001 (2004).

[9] K. Singer, M. Reetz-Lamour, T. Amthor, L. Marcassa, and M. Weidemüller, Phys. Rev. Lett. 93, 163001 (2004).

[10] D. Jaksch, J. I. Cirac, P. Zoller, S. L. Rolston, R. Côté, and M. Lukin, Phys. Rev. Lett. 85, 2208 (2000).

[11] M. D. Lukin, M. Fleischhauer, R. Côté, L. M. Duan, D. Jaksch, J. Cirac, and P. Zoller, Phys. Rev. Lett. 87, 037901 (2001).

[12] S. D. Gensemer and P. L. Gould, Phys. Rev. Lett. 80, 936 (1998).

[13] M. Wright, S. Gensemer, J. Vala, R. Kosloff, and P. Gould, Phys. Rev. Lett. 95, 063001 (2005).

[14] W. Li, P. J. Tanner, and T. F. Gallagher, Phys. Rev. Lett. 94, 173001 (2005).

[15] K. Singer, J. Stanojevic, M. Weidemüller, and R. Côté, J. Phys. B: At. Mol. Opt. Phys. 38, S295 (2005). 
[16] C. Boisseau, I. Simbotin, and R. Côté, Phys. Rev. Lett. 88, 133004 (2002).

[17] K. Singer, M. Reetz-Lamour, T. Amthor, S. Fölling, M. Tscherneck, and M. Weidemüller, J. Phys. B 38, S321 (2005).

[18] A. Gallagher and D. E. Pritchard, Phys. Rev. Lett. 63, 957 (1989).
[19] A. Walz-Flannigan, J. R. Guest, J.-H. Choi, and G. Raithel, Phys. Rev. A 69, 063405 (2004).

[20] T. F. Gallagher, Rydberg Atoms (Cambridge University Press, Cambridge, 1994).

[21] T. F. Gallagher, priv. comm. 